\title{
The Alleviating Pain Effect of Aqueous Extract from Tong-Xie-Yao-Fang, on Experimental Visceral Hypersensitivity and Its Mechanism
}

\author{
Xu-Guang Hu, ${ }^{a, b}$ Di Xu ${ }^{a, c}$ Yue Zhao, ${ }^{a}$ Xiang-Bo Yang,${ }^{b}$ Jiang Meng, ${ }^{a, b}$ Han Shen, ${ }^{a, b}$ and Jiao Guo ${ }^{*, a}$ \\ ${ }^{a}$ Guangdong Pharmaceutical University; Guangzhou 510006, P. R. China: ${ }^{b}$ MOE Key Laboratory of Laser Life Science \\ and Institute of Laser Life Science, South China Normal University; Guangzhou 510631, P. R. China: and ${ }^{c}$ South China \\ Agricultural University; Guangzhou 510642, P. R. China. \\ Received November 12, 2008; accepted February 18, 2009; published online March 27, 2009
}

\begin{abstract}
Tong-Xie-Yao-Fang (TXYF) is a prescription in traditional chinese medicine (TCM), used for relieving abdominal pain associated with irritable bowel syndrome. The aim of the present study was to investigate the effects and mechanism of TXYF on experimental visceral hypersensitivity (VH) models. TXYF affected the abdominal withdrawal reflex produced by colonic distention in maternal separation-induced visceral hypersensitivity rats, in a dosage-dependent manner. TXYF significantly decreased serotonin (5-HT) levels in serum and corticotrophin releasing factor (CRF) concentrations in the brain. Moreover, it was found that VH alleviation by TXYF was dependent on the substance $P$ (SP) expression in the colon mucosa. These results suggest that TXYF attenuates behavioral hyperalgesia by regulating substance associated with the brain-gut axis, including decreasing the expression of 5-HT and SP in the periphery and that of CRF in the center.
\end{abstract}

Key words visceral hypersensitivity; Tong-Xie-Yao-Fang; serotonin; corticotrophin-releasing factor; substance P

Irritable bowel syndrome (IBS) is a common digestive disease characterized by abdominal discomfort or pain associated with disturbance in bowel habits. Although the precise mechanism of IBS has not been fully elucidated, dysfunction of the bidirectional communication between the gut and the brain (the brain-gut axis) has the most significant impact on IBS. ${ }^{1)}$

Visceral hypersensitivity (VH) is an important characteristic feature of IBS, inflammatory bowel disease and other functional bowel disorders. ${ }^{2}$ It is thought to be a pain state caused by central and peripheral sensitization, which leads to abnormal perception both of painful and non-painful stimuli. ${ }^{3)}$ Long-lasting pain states, chronic inflammation, genetic factors and many unknown factors contribute to the generation of $\mathrm{VH}$.

Tong-Xie-Yao-Fang (TXYF), a prescription in traditional chinese medicine (TCM), has been widely used to relieve symptoms associated with IBS for a long time. This prescription contains white atractylodes rhizome, white peony root, dried old orange peel and ledebouriella root. $\left.{ }^{4}\right)$ TXYF has been believed to be effective in improving disorders of the digestive system, alleviating abdomen pain, diarrhea and widely used as a medication to treat IBS and ulcerative colitis (UC) clinically. ${ }^{5-7)}$ However, as for most other traditional prescriptions, the mechanism of abdomen pain alleviation of TXYF is still not clear. The present study was planned to investigate the effects of TXYF on a maternal separation-induced VH model and its mechanism of action.

\section{MATERIALS AND METHODS}

Animals Primiparous timed-pregnant Sprague-Dawley rats on gestational days 15-16 were obtained from the Guangdong Experimental Animal Center. Six dams were individually housed in cages containing bedding material on a $12: 12$-h light-dark cycle (lights on at 08:00) and provided with food and water ad libitum. All studies were performed in accordance with the proposals of the Committee for Research and Ethical Issues of the International Association and were approved by the Committee on the Use of Human and Animal Subjects in Teaching and Research, Guangdong Pharmaceutical University.

Preparation of Tong-Xie-Yao-Fang The composition of TXYF used in this study was in fixed proportion: white atractylodes rhizome $200 \mathrm{~g}$, white peony root $200 \mathrm{~g}$, dried old orange peel $150 \mathrm{~g}$ and ledebouriella root $100 \mathrm{~g}$. These crude herbs were purchased from Zhixin Pharmaceutical Factory (Guangzhou, China) and identified by Professor Shu-yuan Li (School of Chinese Medicine, Guangdong Pharmaceutical University). TXYF was prepared by mixing the four crude drugs. TXYF $(650 \mathrm{~g})$ was ground and soaked in $6500 \mathrm{ml}$ of water for $30 \mathrm{~min}$, then refluxed twice for $1 \mathrm{~h}$ each. Water was evaporated under reduced pressure, and the extracts were dried to powder with $12.2 \%$ yield $(\mathrm{w} / \mathrm{w})$. The extracts were freshly prepared with distilled water at the desired concentrations just before use. Aqueous extracts of TXYF $2.4 \mathrm{~g} / \mathrm{kg}$ and $1.2 \mathrm{~g} / \mathrm{kg}$ were defined as high dosage and low dosage, respectively.

Chemicals Chemical reagents used in this study were: alosetron and serotonin (Sigma, U.S.A.), diethyl ether and paraformaldehyde of analytical grade (Guangzhou Chemical Reagents, China), and hydrate chloride of analytical grade (Yuanhang Chemical Factory, China). The corticotrophinreleasing factor measurement kit was obtained from Jiangcheng Bioengineering Institute (Nanjing, China).

Maternal Separation Protocol Forty rat pups were separated from their dams from postnatal days 8 to 21 , and 10 rat pups in the normal group remained with their mothers. Separated pups were removed from their cages and dams on postnatal days $8-21$ for 180 min daily by placing them in individual compartments within plastic cages, in an adjacent room to their home cages. The new cages were lined with chip bedding and kept at $37^{\circ} \mathrm{C}$ using a heating pad placed underneath the cages. Maternal Separation (MS) was done at 09:00 each day to minimize the effects of circadian rhythm. Normal pups were not handled but maintained in their home cages with their dams until they were weaned. Litters were culled to 8 pups per dam on postnatal day 7 to ensure even 
litter sizes and maternal care. On postnatal day 23, the pups' sex was determined, and they were weaned and housed in individual cages. After weaning, the pups were weighed weekly (data not shown). Only males were utilized in this study to avoid variations due to hormonal cycling.

Examination of Abdominal Withdrawal Reflex Examination of the relative abdominal withdrawal reflex (AWR) was measured using the procedure modified from previous reports. ${ }^{8)}$ Briefly, rats were lightly anesthetized with diethyl ether, and a balloon $(4 \mathrm{~cm}$ in length, made from the finger of a latex glove) was inserted through the anus into the rectum and descending colon, attached to a Fogarty catheter. The open end of the balloon was secured to the catheter with thread and wrapped with tape $(1 \mathrm{~cm}$ wide). Prior to use, the balloon was inflated and left overnight so that the latex could stretch and the balloon could become compliant. The balloon was inserted so that the thread was approximately $1 \mathrm{~cm}$ proximal to the anal sphincter, and was held in place by taping the tubing to the tail. The catheter was attached via a connector to a sphygmomanometer pump and a pressure gauge. The rats were then housed in small Lucite cubicle $(20 \times 8 \times 8 \mathrm{~cm})$ on an elevated plexiglas platform and allowed to wake up and adapt for $30 \mathrm{~min}$. Colorental distention (CRD) was produced by rapidly inflating the balloon to the desired pressure $(20,40,60$, or $80 \mathrm{mmHg})$ for a duration of $10 \mathrm{~s}$. Stimuli were applied in an ascending graded manner (spaced by $4 \mathrm{~min}$ ). To decrease the "human factor" bias to the minimum possible, the same stimulation paradigm was used in every rat. The AWR, an involuntary motor reflex similar to the visceromotor reflex, was recorded. The AWR score was assigned as follows: $0=$ no behavioral response to distension, $1=$ brief head movements followed by abdominal muscle without immobility, $2=$ contraction of lifting of abdomen, $3=$ lifting of abdomen, $4=$ body arching and lifting of pelvic structure. Measurements of the AWR by visual observation were reproduced by two blinded observers. The pain threshold pressure (PTP) is defined as the stimulus pressure that evokes a visually identifiable contraction of the abdominal wall. Increasing pressure was applied in steps of $5 \mathrm{mmHg}$ lasting $30 \mathrm{~s}$ until pain behavior was displayed or until $80 \mathrm{mmHg}$ was reached to avoid lasting damage to the animals.

Detection of 5-HT in Serum 5-HT levels in serum were assayed with the procedure modified from a previous report. ${ }^{9)}$ Briefly, the serum was mixed with $5 \mathrm{ml}$ of acidified butanol ( $\mathrm{HCl} 850 \mu \mathrm{l}$ in 11 butanol) in a glass tube by a motor driven pestle (Ultra-Turrax T8, IKA-Werke, Germany). The volume of each homogenate was adjusted to $25 \mathrm{ml}$ and centrifuged at $4{ }^{\circ} \mathrm{C}$ and $3000 \times \boldsymbol{g}$ for $10 \mathrm{~min}$. A $3 \mathrm{ml}$ aliquot of each supernatant was transferred to a tube containing $5 \mathrm{ml}$ of $n$-heptane and $600 \mu \mathrm{l}$ of acidic cysteine $(0.1 \% \mathrm{w} / \mathrm{v}$ in $\mathrm{HCl}$ $0.1 \mathrm{M}$ ); the contents of each tube were mixed for $2 \mathrm{~min}$ at $4{ }^{\circ} \mathrm{C}$ and centrifuged at $3000 \times \boldsymbol{g}$ for $5 \mathrm{~min}$. From each tube the upper organic phase together with the disc at the organic/ aqueous inter-phase was removed by aspiration and discarded. A $200 \mu \mathrm{l}$ aliquot of the lower aqueous phase with $20 \mu \mathrm{l}$ of $1 \%$ cysteine and $800 \mu \mathrm{l}$ of HCL containing $0.004 \%$ $o$-phthalaldehyde was incubated at $77^{\circ} \mathrm{C}$ for $15 \mathrm{~min}$. When the tube was cooled to room temperature, the fluorescence was measured in an Aminco-Bowman spectrophotofluorimeter at an activation wavelength of $365 \mathrm{~nm}$ and emission wave length $470 \mathrm{~nm}$. Standards were prepared by dissolving 5-HT in the distilled water so that $200 \mu \mathrm{l}$ volumes containing 5 -HT $50-200 \mathrm{ng}$ could be added to the serum to serve as the internal standards. These were prepared throughout the assay procedure.

Measurement of Corticotrophin Releasing Factor (CRF) in Brain Tissue Rat brain tissue was dissected and about $0.4 \mathrm{~g}$ of brain tissue from hypothalamus was stored at $-20^{\circ} \mathrm{C}$. The brain tissue was thawed on a chilled glass plate $16 \mathrm{~h}$ later. The CRF was extracted from dissected tissue in $\mathrm{HCl} 1 \mathrm{M}$ with protease inhibitors as previously described. ${ }^{10)}$ The CRF concentration was determined using a CRF radioimmunoassay assay kit according to the manufacturer's protocol (HuaYing Biotechnology Institute of Beijing).

Substance P Imunohistochemical Staining The colon samples were embedded in paraffin in the usual manner and stained with an immunohistochemical method. Rabbit antihuman substance P antibody (Serotec Company, U.S.A., product No. 2025R214, dilution 1:1000) was used as the primary antibody. The secondary antibody staining kit was purchased from JingMei Company (Beijing, China). Then, the samples were observed under a powerful optical microscope $(\times 400)$. Each piece of colon was observed continuously from six non-overlapping fields of view, and the numbers of immunoreactive-positive cells were counted.

Statistical Analysis Results are expressed as mean S.D. The effect of TXYF on each parameter was examined using one-way analysis of variance. Individual differences among groups were analyzed using Dunnett's test and differences of $p<0.05$ were considered significant.

\section{RESULTS}

Effects of TXYF on MS-Induced Colon Hypersensitivity in VH Rats On postnatal days 63, TXYF at high and low dosage and alosetron (5- $\mathrm{HT}_{3}$ antagonist) $150 \mathrm{mg} / \mathrm{kg}$ were administered orally for $14 \mathrm{~d}$. One hour after the final administration, the relative AWR was measured following the procedure modified from previous reports. ${ }^{8)}$

As shown in Fig. 1a, the AWR scores in response to graded CRD $(20,40,60,80 \mathrm{mmHg})$ were $0,1.2 \pm 0.3,3 \pm 0.3$ and $3.4 \pm 0.2$ in the normal group, and $0.2 \pm 0.1,3 \pm 0.3$, $3.2 \pm 0.2$, and $3.7 \pm 0.3$ in the VH group. The PTP in response to $\mathrm{CRD}$ was $53.2 \pm 6.0 \mathrm{mmHg}$ in the normal group and $37.4 \pm 3.5 \mathrm{mmHg}$ in the VH group (Fig. 1b). Thus, there was a significant difference in rectal sensitivity to CRD between the Normal control group and VH model group. These results suggest that the VH model exhibited visceral hypersensitivity.

TXYF at high and low dosage significantly elevated the PTP and reduced AWR scores in VH rats in a dose-dependent manner. AWR scores in response to graded CRD (20, 40, $60,80 \mathrm{mmHg}$ ) were $0,0.2 \pm 0.2,2.1 \pm 0.2$, and $2.5 \pm 0.2$ and 0 , $0.4 \pm 0.2,2.4 \pm 0.2$, and $2.8 \pm 0.2$ after the high- and low-dose TXYF treatment, respectively (Fig. 1a). The PTP in response to $\mathrm{CRD}$ was $49.8 \pm 3.5 \mathrm{mmHg}$ and $47.3 \pm 4.2 \mathrm{mmHg}$ after the high and low dosage of TXYF treatment, respectively (Fig. 1b). These results suggest that TXYF has analgesic effects on $\mathrm{VH}$ rats like Alosetron.

Effects of TXYF on Serum 5-HT in VH Rats After the AWR score and the threshold pressure of rectal distension were measured, the rats inhaled aether for anaesthesia and 
$5 \mathrm{ml}$ of blood was drawn from the suborbital vein. The blood samples were kept in a refrigerator at $4{ }^{\circ} \mathrm{C}$ for $2 \mathrm{~h}$ and then centrifuged at $2800 \times \boldsymbol{g}$ for $10 \mathrm{~min}$. The serum was prepared from the superstratum.
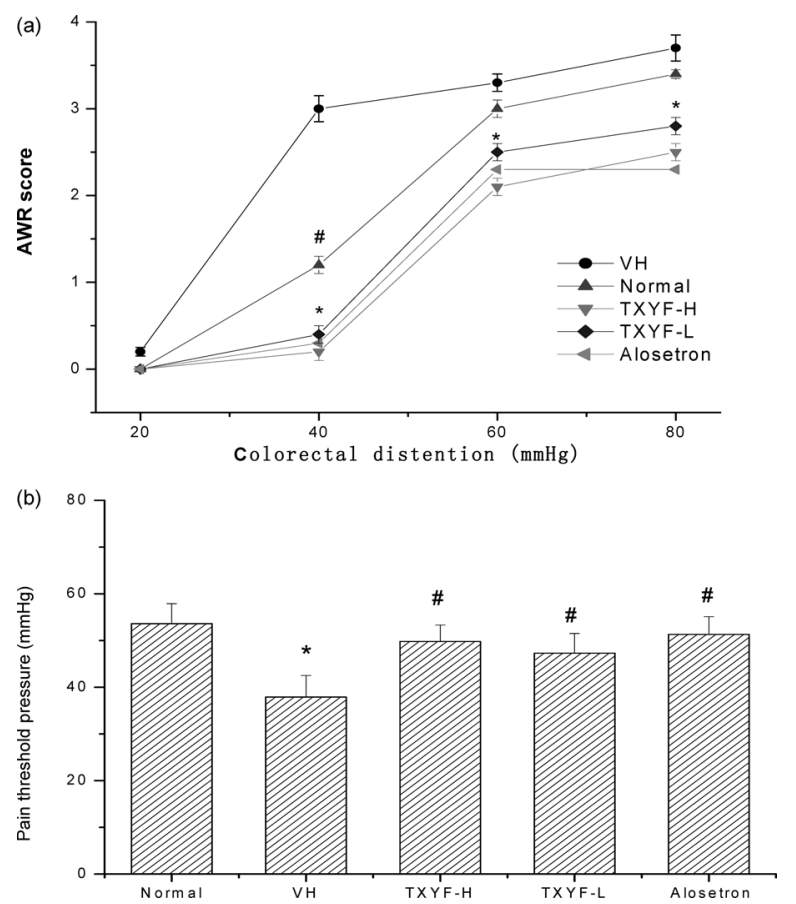

Fig. 1. (a) Abdominal Withdrawal Reflex Score (AWR Score) Measured in Response to Graded Colorectal Distension Shows Significant Differences between the Rats with Visceral Hypersensitivity and the Normal Control Groups at $40 \mathrm{mmHg}$, and between the TXYF Treatment Group and the VH Group at 40,60 and $80 \mathrm{mmHg}$

Data are mean \pm S.D. $\# p<0.05 v s$. normal, $* p<0.05 v$ s. VH.

(b) Pain Threshold Pressure (PTP) Measured in Response to Colorectal Distension Showed Significant Decrease in the Rats with Visceral Hypersensitivity Comparing to the Normal Control Group, and a Significant Increase in the TXYF Treatment Group Comparing to the VH Group

Data are mean \pm S.D. $* p<0.05 v s$. normal, $\# p<0.05 v s$. VH.
As shown in Table 1, the 5-HT level in serum was higher in $\mathrm{VH}$ rats than in normal rats $(2456 \pm 211 \mathrm{ng} / \mathrm{ml} v s$. $1572 \pm$ $232 \mathrm{ng} / \mathrm{ml}$ ), while TXYF significantly decreased 5-HT level in a dose-dependent manner. After treatment with high- or low-dose TXYF, the 5-HT levels decreased to 1786 \pm 165 and $1854 \pm 189 \mathrm{ng} / \mathrm{ml}$, respectively. These results suggest that TXYF has analgesic effects on VH rats in a dose-dependent manner through decreasing the serum level of 5-HT.

Effects of TXYF on Brain CRF in VH Rats After collecting the blood samples, the rats were sacrificed and the brain tissues were collected. As shown in Table 2, the CRF concentration in the brain was higher in $\mathrm{VH}$ rats than in normal rats $(254 \pm 25 \mathrm{pg} / \mathrm{mg}$ vs. $98 \pm 22 \mathrm{pg} / \mathrm{mg})$. TXYF significantly decreased CRF levels in a dose-dependent manner. After high- and low-dose TXYF treatment, the CRF concentration decreased to $116 \pm 16$ and $135 \pm 18 \mathrm{pg} / \mathrm{mg}$, respectively. These results suggest TXYF has analgesic effects on

Table 1. The Effect of TXYF on 5-HT Content in Serum

\begin{tabular}{lccl}
\hline \multicolumn{1}{c}{ Group } & Dose $(\mathrm{g} / \mathrm{kg})$ & No. of rats & 5-HT (ng/ml) \\
\hline Normal rats & - & 10 & $1572 \pm 232$ \\
VH rats control & - & 10 & $2456 \pm 211^{* *}$ \\
TXYF-H rats & 2.4 & 10 & $1786 \pm 165^{\#}$ \\
TXYF-L rats & 1.2 & 10 & $1854 \pm 189^{\#}$ \\
Alosetron rats & $1.5 \times 10^{-3}$ & 10 & $1745 \pm 156^{\#}$ \\
\hline
\end{tabular}

$* * p<0.01$ vs. normal rats; $\# p<0.05$ vs. VH rats control.

Table 2. The Effect of TXYF on CRF Content in Brain

\begin{tabular}{lccc}
\hline \hline \multicolumn{1}{c}{ Group } & Dose $(\mathrm{mg} / \mathrm{kg})$ & No. of rats & CRF (pg/mg) \\
\hline Normal rats & - & 10 & $98 \pm 22$ \\
VH rats control & - & 10 & $254 \pm 25^{* *}$ \\
TXYF-H rats & 2.4 & 10 & $116 \pm 16^{\#}$ \\
TXYF-L rats & 1.2 & 10 & $135 \pm 18^{\#}$ \\
Alosetron rats & $1.5 \times 10^{-3}$ & 10 & $195 \pm 21^{\#}$ \\
\hline
\end{tabular}

$* * p<0.01$ vs. normal rats; $\# p<0.05 v s$. VH rats control.

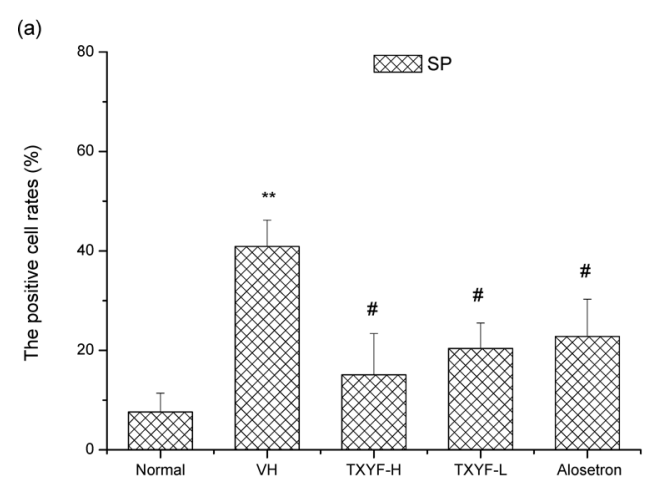

Fig. 2. (a) The Positive Cells Rates of SP Expression in the Colon Mucosa in 5 Groups Involving Normal Group, VH Group, TXYF-Low Dosage Group, TXYF-High Dosage Group and Alosetron Group Showed That VH Group Had Higher Expression of SP Than Normal Group, and TXYF-Low Dosage Group, TXYF-High Dosage Group All Decreased the SP Expression in VH Rats

Data are mean \pm S.D. $* * p<0.01 v s$. normal, $\# p<0.05 v s$. VH.

(b) Photomicrograph Findings of SP Expression in Colon Mucosa of Rats

There was a significant increase in SP expression in the colon mucosa of animals with VH, comparing with normal rats. TXYF significantly alleviated the expression of SP in rats with $\mathrm{VH}(\times 400)$.

(b)

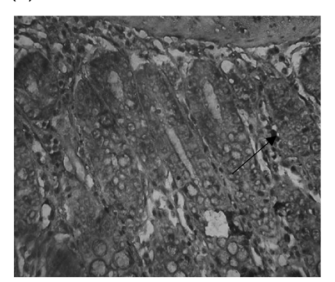

Normal group

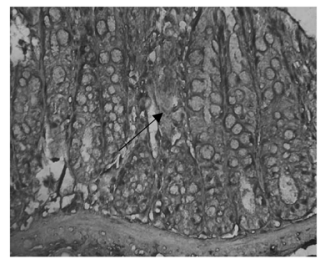

TXYF-High dosage group

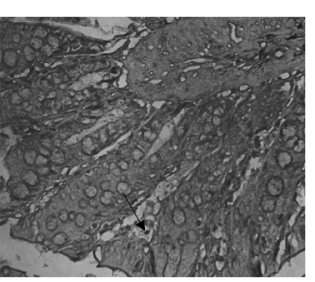

VH group

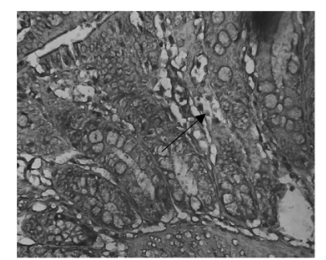

Alosetron group

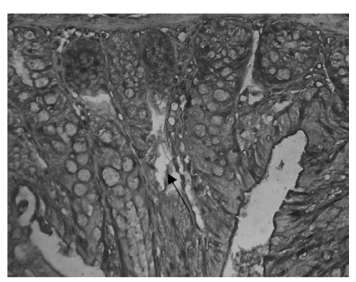

TXYF-Low dosage group 
$\mathrm{VH}$ rats in a dose-dependent manner by reducing the level of CRF, similar to alosetron.

Effects of TXYF on SP Expression in the Colonic Mucosa of VH Rats A $2 \mathrm{~cm}$ colon section was dissected and post-fixed in formalin solution overnight. The mucosa samples were embedded in paraffin in the usual manner and stained with immunohistochemical methods.

As shown in Figs. 2a and b, quantification of SP expression by counting the positive cell rates in the colonic mucosa revealed that rats undergoing MS had higher levels of SP expression $(40.9 \pm 5.3 \%)$ than that of normal rats $(7.6 \pm 3.8 \%)$. After high- and low-dose TXYF treatment, expression of SP in the colonic mucosa decreased to $15.1 \pm 8.3 \%$ and $20.4 \pm$ $5.1 \%$, respectively. The positive rate of SP in colonic mucosa was significantly reduced by the administration of both TXYF and alosetron.

\section{DISCUSSION}

The present study examined the effects of TXYF on MSinduced VH rats. Fourteen-day administration of the prescription significantly increased the pain threshold and decreased the AWR score in a dose-dependent manner. These results suggest that TXYF attenuates behavioral hyperalgesia in VH model rats. Clinical observations revealed that IBS involved multiple factors, such as mental, immune, and social factors. ${ }^{11,12)}$

Basic research related to the mechanisms of chronic visceral pain has attracted more attention in the last decade. ${ }^{13}$ ) At present, it is considered that $\mathrm{VH}$ results from long-lasting pain states, chronic inflammation, genetic factors, and molecular messenger mediators, such as 5-HT, calcitonin generelated peptide (CGRP), SP, etc. ${ }^{14)}$ The strong link between the central nervous system (CNS) and the enteric nervous system (ENS) which develops $\mathrm{VH}$ is also confirmed by the fact that some neuropeptides and receptors are present in both the ENS and the CNS. Putative agents include enkephalins, SP, CGRP, thyrotrophin releasing hormone (TRH), CRF, 5-HT, oxytocine, vasopressin, somatostatin, and cholecystokinin (CCK). ${ }^{15-17)}$ Previous studies showed that 5HT plays a major role in the modulation of brain-gut communication and in VH via activation of secretory cells, stimulation of afferent and efferent neurons and direct effects on gut smooth muscle, leading to contraction or relaxation. ${ }^{18)}$ The majority of the body's 5-HT is stored in the enterochromaffin cells that are scattered in the epithelium of the intestine and colon, while a minority is distributed in the myenteric neurons that serve as neurotransmitters of the ENS, synapsing with the ascending and descending interneurons, resulting in circular muscle contraction and relaxation to initiate the peristaltic reflex, associated with the releasing of SP, CGRP, nitric oxide (NO), vasoactive intestinal peptide, ATP, etc. ${ }^{19-21)}$

Major studies of 5-HT levels in the colonic mucosa and serum of IBS patients led to different conclusions. Most found that 5-HT in the colonic mucosa and serum increased in IBS patients. ${ }^{22)}$ For example, Moskwa et al. ${ }^{23)}$ found that 5-HT serum levels in patients with IBS-C and IBS-D were significantly higher than in the healthy group. Simon et al. ${ }^{24)}$ reported that 5-HT in the plasma and colonic mucosa in IBSD significantly increased and the 5-HT increase in the plasma in IBS-C patients was higher than in healthy volunteers. A study conducted by Wang et al. ${ }^{25)}$ in China suggested that IBS-C patients had low levels of 5-HT in various parts of the intestinal mucosa. Coates et al. $^{26)}$ confirmed that the 5-HT levels in the rectal mucosa in patients with IBS were significantly decreased compared with the control group. Simren et al. $^{27)}$ showed that 5-HT levels in the plasma of IBS-D patients were significantly lower than in healthy volunteers.

Our study found that 5-HT levels and SP expression in VH rats were significantly higher than in normal rats. These results indicate that 5-HT and SP in the periphery are involved in the mechanism of VH in the rat model. After TXYF treatment, 5-HT levels and SP expression decreased while the pain threshold in $\mathrm{VH}$ rats increased.

Experimental evidence suggests that MS stress-induced $\mathrm{VH}$ involves CRF, which is released by the hypothalamus during stress and activates the hypothalamic-pituitiary-adrenal axis (HPA) to produce the stress hormone cortisol. In animal models, neonatal adversity can result in permanent functional changes in the stress-mediating systems of the CNS. Newborn rats subjected to MS demonstrate increased release of corticotropin-releasing hormone $(\mathrm{CRH})$, altered expression of glucocorticoid receptors, as well as changes in the norepinephrine and GABA systems. ${ }^{28)}$ Recently, studies have shown that humans experiencing adverse early-life events were associated with responsiveness to stress and alterations in the HPA axis. There is evidence that early-life trauma and ongoing psychological stress can affect the clinical course of intestinal disorders. ${ }^{29)}$ Rats exposed to neonatal trauma in the form of MS showed visceral hyperalgesia and increased colonic motility in response to stress at 2 months of age. ${ }^{30)}$ Bayati et al. ${ }^{31)}$ demonstrated that local manipulation of the colon in neonatal rats induced chronic VH to painful stimuli. Thus neonatal trauma can alter colonic neuromuscular function in adult rats. In rats, central administration of CRF mimics the effect of restraint stress in increasing the number of abdominal contractions in response to rectal distension. ${ }^{32}$ Our findings suggest that the CRF concentration in the brain is increased in MS-induced $\mathrm{VH}$ rats, which indicates that $\mathrm{CRF}$ in the center is involved in the mechanism of $\mathrm{VH}$ in the rat model. After TXYF treatment, the CRF concentration decreased and the pain threshold of VH rats increased.

Previous research showed that TXYF can inhibit neonatal colon irritation-induced VH by decreasing 5-HT in the serum and decreasing SP and CGRP in the plasma. ${ }^{33)}$ TXYF can treat IBS probably by affecting the secretion and release of gastrointestinal hormone (VIP) after the partial restriction stress binding stimulation in the IBS rat model. ${ }^{34)}$ TXYF has a marked inhibitory effect on the degranulation of peritoneal mast cells in sensitized rats induced with C48/80, which indicates that TXYF exerts therapeutic effect by inhibiting enteric mast cell activation and thus decreasing histamine release, which in part explains the mechanism of TXYF in IBS. $^{35)}$ TXYF can inhibit intestinal muscle contraction activation by antagonizing $\mathrm{M}$ receptors. ${ }^{36)}$

Overall, our present findings provide evidence that TXYT attenuates behavioral hyperalgesia in $\mathrm{VH}$ rats by regulating substances related to the brain-gut axis, including decreasing 5-HT and SP expression in the periphery, alleviating CRF in the center. These findings confirm that TXYT is a useful pre- 
scription for the treatment of abdominal pain in IBS. More thorough investigation is required to elucidate the clinical implications.

Acknowledgments This work was supported by the MOE Key Laboratory of Laser Life Science and Institute of Laser Life Science (South China Normal University, Guangzhou, P. R. China).

\section{REFERENCES}

1) Boivin M., Bouin M., Plourde V.. Gastroenterology, 122, 1771-1777 (2002).

2) Delvaux M., Gut, 51, 67-71 (2002).

3) Mayer E. A., Gebhart G. F., Gastroenterology, 107, 271-293 (1994).

4) Fan H., Qiu M. Y., Mei J. J., Shen G. X., Liu S. L., Chen R., World J. Gastroenterol., 11, 4800-4806 (2005).

5) Ye L., Zhejiang J. TCM, 10, 60-62 (2000).

6) Chen J., Bian T., JiangSu J. TCM, 8, 12-14 (2005).

7) Bian Z., Wu T., Liu L., Miao J., Wong H., Song L., Sung J., J. Altern. Complement. Med., 12, 401-407 (2006).

8) Al-Chaer E. D., Kawasaki M., Pasricha P. J., Gastroenterology, 119, 1276 - 1285 (2000).

9) Farmer S. G., Laniyonu A. A., Br. J. Pharmacol., 82, 883-889 (1984).

10) Stout S., Owens M., Charles B., J. Pharmacol. Exp. Ther, 300, 115120 (2003).

11) Mach T., Med. Sci. Monit., 10, 125-131 (2004).

12) Thompson W. G., Longstreth G. F., Drossman D. A., Heaton K. W., Irvine E. J., Muller-Lissner S. A., Gut, 45, 43-47 (1999).

13) Bueno L., Fioramonti J., Delvaux M., Frexinos J., Gastroenterology, 112, 1714-1743 (1997).

14) Mertz H., Aliment. Pharmacol. Ther., 17, 623-633 (2003).

15) Wood J. D., Alpers D. H., Andrews P. L. R., J. Altern. Complement. Med., 13, 245-250 (2005).

16) Yew D., Ziea E., "Inter Medicine," McLean, Degon and Associates,
London, 2000, pp. 31-40.

17) Mulak A., Bonaz B., Med. Sci. Monit., 10, 55-62 (2004).

18) Kim D. Y., Camilleri M., Am. J. Gastroenterol., 95, 2698-2709 (2000).

19) Crowell M. D., Br. J. Pharmacol., 141, 1285-1293 (2004).

20) Wang Z. J., Ouyang Q., Zou Y. G., Peng L., Wang Y. F., Zhonghua Neike Zazhi, 11, 851-852 (2005).

21) Pan H., Gershon M. D., J. Neurosci., 20, 3295-3309 (2000).

22) Miwa J., Echizen H., Matsueda K., Umeda N., Digestion, 63, 188 194 (2001).

23) Moskwa A., Chojnacki J., Stec-Michalska K., Szadkowski N., Pol. Merkur. Lekarski., 131, 366-368 (2007).

24) Simon P., David J., Robin C., Gastroenterology, 125, 1651-1653 (2003).

25) Wang S. H., Lei D., Luo J. Y., Jun G., Li L., Lu X. L., Han S. P., World J. Gastroenterol., 45, 6041-6047 (2007).

26) Coates M. D., Mahoney C. R., Linden D. R., Sampson J. E., Chen J., Blaszyk H., Gastroenterology, 126 , 1657-1664 (2004).

27) Simren M., Abrahamsson H., Biornson E. S., Gut, 48, $20-29$ (2001).

28) Soderholm J. D., Yates D. A., Gareau M. G., Yang P. C., MacQueen G., Perdue M. H., Am. J. Physiol. Gastrointest. Liver Physiol., 283, $1257-1263$ (2002).

29) Anisman H., Zaharia M. D., Meaney M. J., Merali Z., Int. J. Dev. Neurosci., 16, 149-164 (1998).

30) Sagami Y., Shimada Y., Tayama J., Gut, 53, 958-964 (2004).

31) Bayati A. I., McRoberts J. A., Mayer E. A., Am. J. Physiol. Gastrointest. Liver Physiol., 1282, 307-316 (2002).

32) Gue M., Del Rio-Lacheze C., Eutamene H., Theodorou V., Fioramonti J., Bueno L., Neurogastroenterol. Motil., 9, 271-279 (1997).

33) Zhao Y. B., Li D. G., Chinese Traditional Patent Medicine, 28, 852 855 (2006).

34) Li D. H., Li C. M., Li W. S., Zhou Z. D., Zhang Y., Lishizhen Medicine and Maternal Medical Research, 9, 2098-2099 (2007).

35) Liu Z. G., Zhao Y. B., Modern J. Integrated Trad. Chin. Western Med., 14, 1901-1903 (2007).

36) Wang P. P., Chu G. W., Zheng M., Zhejiang J. TCM, 4, 235-237 (2007). 